\title{
Flowcharting the Meaning of Logic Formulas
}

\author{
Sabah Al-Fedaghi \\ Computer Engineering Department \\ Kuwait University \\ Kuwait
}

\begin{abstract}
In logic, representation of a domain (e.g., physical reality) comprises the things its expressions (formulas) refer to and their relationships. Recent research has examined the realm of nonsymbolic representations, especially diagrams. It is claimed that, in general, diagrams have advantages over linguistic descriptions. Current diagrammatic representations of logic formulas do not completely depict their underlying semantics, and they lack a basic static structure that incorporates elementary dynamic features, creating a conceptual gap that can lead to misinterpretation. This paper demonstrates a methodology for mapping the sense of a logic formula and producing diagrams that integrate linguistic conception, truthvalues, and meaning and can be used in teaching, communication, and understanding, especially with students specializing in logic representation and reasoning.
\end{abstract}

Keywords-knowledge representation; logic formulas; diagrammatic representation; sense

\section{INTRODUCTION}

In computer science, studies suggest that flowcharts have potential as tools in educational settings [1-3], despite the fact that complaints regarding their value in design and education [4-5] have led to their near-elimination since the mid-1970s. Currently (2016), flowcharts are "still very common in procedure documentation [and] some managers love them and use them for everything" [6]. They have also been revived in the form of UML activity diagrams. "Recently, many philosophers, psychologists, logicians, mathematicians, and computer scientists have become increasingly aware of the importance of multi-modal reasoning and, moreover, much research has been undertaken in the area of non-symbolic, especially diagrammatic, representation systems" [7]. Accordingly, any development of diagrammatic language is a contribution in this direction.

In computer science, flowcharting is useful as a form of program documentation, as a means of enhancing algorithm understanding and design, and as a tool for teaching programming [1]. In the field of traditional logic diagrams, studies of relationships between diagrammatic and linguistic representation systems have found advantages in diagrammatic representations over linguistic ones [8-9]. Venn diagrams, Euler circles, and Peirce's existential graphs are examples of these representations.

Certainly, in their proper form, flowcharts and diagrams can be used to represent a logic domain (e.g., physical reality, mathematics) that comprises the things its expressions (formulas) refer to and their relationships. The advantages in this context include the following:
- Teaching: Among the many applications of diagrams in an educational setting, they are especially useful for students specializing in logic representation and reasoning.

- Communication: Diagrams probably rank among the oldest forms of human communication [7]; they are basic, elementary, and require no spoken language.

- Understanding: Many scientific fields use diagrams to represent or depict knowledge and to assist in understanding of problems [10-13].

Diagrams are used to represent conceptualizations (e.g., underlying deep structures) of language expressions; however, while knowledge embedded in logic formulas can be shown in diagrams, dynamic features are conceptualized (e.g., deduction system, humans) in a way that fails to integrate structure and dynamic features. "It is a quite recent movement among philosophers, logicians, cognitive scientists and computer scientists to focus on different types of representation systems, and much research has been focused on diagrammatic representation systems in particular" [7].

\section{A. Problem}

Current diagrammatic representations of logic formulas do not completely depict their underlying semantics or provide a clear, basic, static structure with elementary dynamic features, creating a conceptual gap that sometimes causes misinterpretation. Structural correspondence between a diagrammatic representation and semantic content "plays a crucial role in both interpretation and inference processes with the representations" [14]

For example, as reported by Shin [15], Venn diagrams lack many features, such as representation of existential statements, but in Euler diagrams, such features as representation of existential statements not only obscure visual clarity but also raise serious interpretational problems. Peirce's diagrams are characterized by arbitrariness in conventions, making them confusing and inconsistent.

\section{B. Proposed solution}

This paper introduces a methodology for expressing the meaning (sense) of a logic formula in a diagram. The resultant schema connects the linguistic version of the formula, the formula's truth values, and its meaning in a diagrammatic apparatus called the Flowthing Model (FM), briefly described in the next section [16]. The example developed in the following sections is a new contribution. 


\section{DIAGRAMMATIC LANGUAGE}

FM uses flowthings to represent a range of things, for example, a logic formula, its meaning, terms, truth values, and so on. Flowthings are defined as what can be created, released, transferred, processed, and received (see Fig. 1; flow is indicated by solid arrows). Hereafter, flowthings are referred to as things. Note that what we call a thing is, in general, not necessarily a substance in the philosophical sense; e.g., heat is a thing that is created, released, transferred, received, and processed.

FM also uses the notions of spheres and subspheres. These are the environments and relationships of the flow. FM also utilizes the notion of triggering, the activation of a flow, denoted in FM diagrams by a dashed arrow.

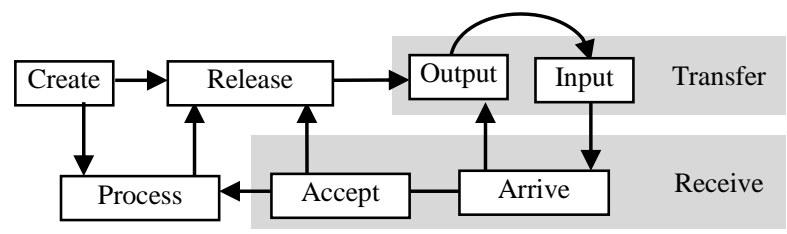

Fig. 1. Flow machine

Example: Borysowich [17] provides a flowchart (not shown here) that

... calculates customer discounts for a company that sells merchandise to wholesale and retail outlets. Wholesale customers receive a two percent discount on all orders. The company also encourages both wholesale and retail customers to pay cash on delivery by offering a two percent discount for this method of payment. Another two percent discount is given on orders of 50 or more units. [17] (Italics added)

Fig. 2 shows the corresponding FM representation of the sequence and flow of these processes.

The company (the global sphere) includes four things in its subspheres: value of the Discount (circle 10), Customer type (2), Payment type (3), and No. of items. Each has its own stream of flow. The procedure for calculating the discount is accomplished as follows.

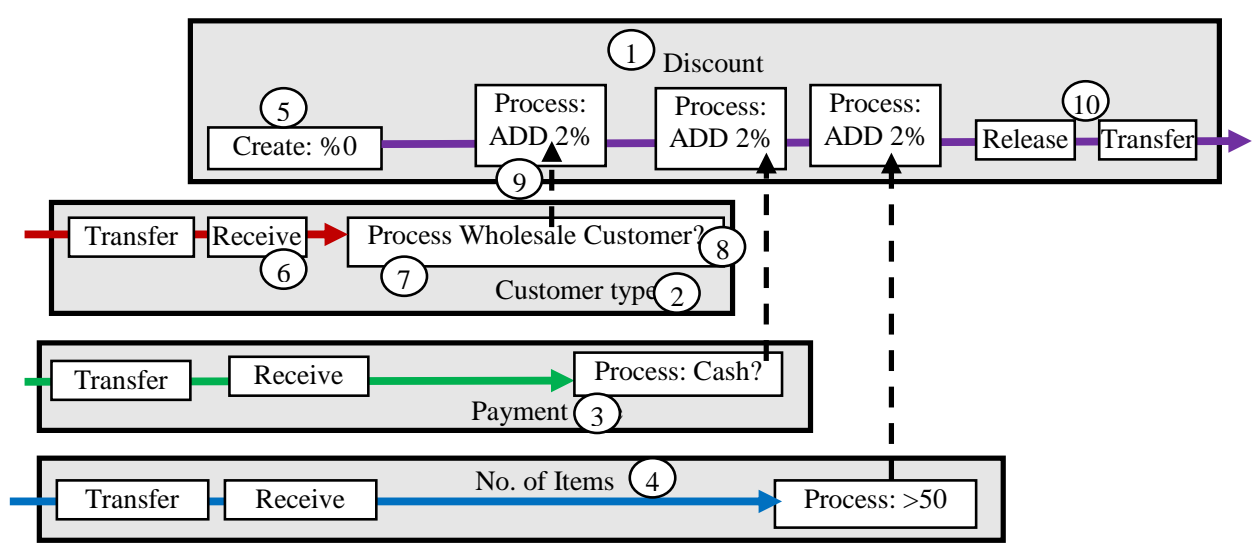

Fig. 2. FM representation of the example

1) The Discount is initiated at $0 \%$ (4). Its flow (movement through the sequence stages) is controlled by triggering from other flows. (This will become clearer later.)

2) The Customer type is received (6) and processed (7); if wholesale, then this triggers (8) adding $2 \%$ to the discount (9). The flow then proceeds along the stream without interruption.

3) A similar procedure is followed for Payment type and then for No. of items.

4) The value of the discount is released and output (10).

Note that, for clarity's sake, Process in Discount is represented by three boxes.

The diagram can be specified in a semiformal language as follows:

Discount.Create $(\% 0)$

Customer Type.Transfer, Receive, Process (If Wholesale Customer: Discount.Process (\%2))
Payment Type.Transfer, Receive, Process (If Cash: Discount.Process (\%2))

No of items.Transfer, Receive, Process (If >50: Discount.Process (\%2))

Discount.Release, Transfer

The familiar dot notation is used to indicate stages inside subspheres. Assuming sequential execution, Fig. 3 shows the path of "control flow" by triggering. In such a sequential execution, it may be necessary to explicitly specify the interruption/resumption of flow inside the Discount sphere. One way to describe this is by storing the intermediate discount value in storage, as shown in Fig. 4. Thus, each internal triggering starts with fetching of the stored value.

It is clear that the FM representation is suitable for identifying parallel execution. For example, using classical function/parameters notation, a rough implementation can be specified as follows: 


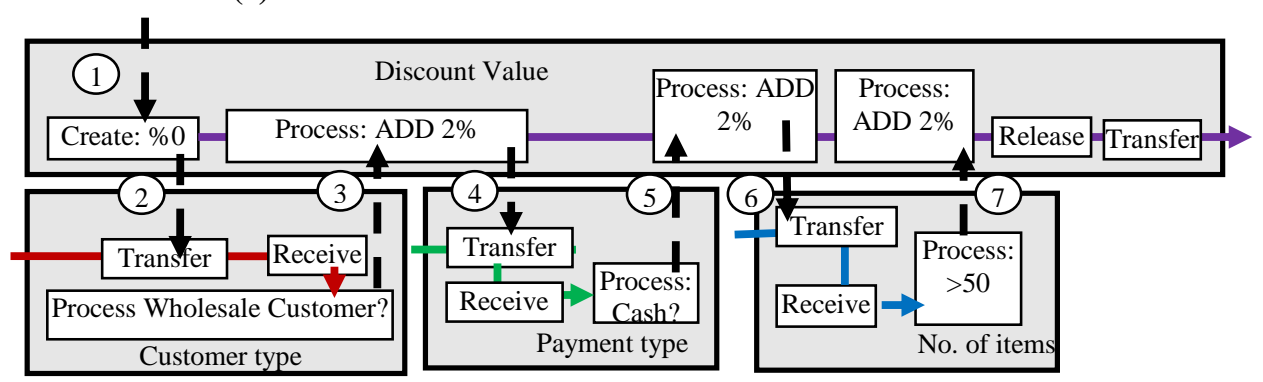

Fig. 3. The sequence of execution of flows or portions of flows

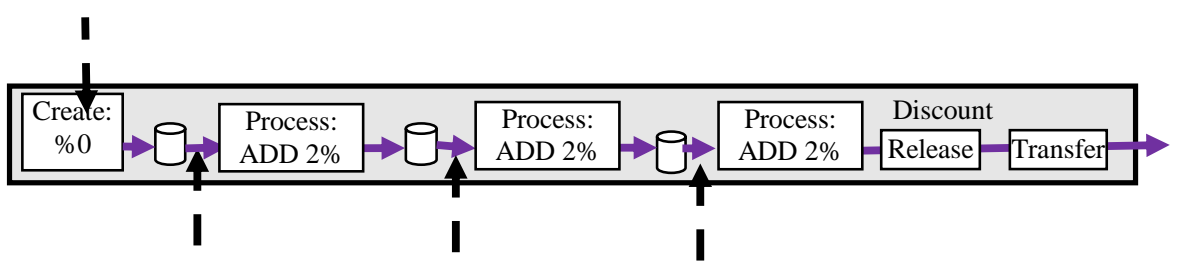

Fig. 4. Explicit description of interruption/resumption of flow

\section{FM AS A LOGIC LANGUAGE}

Current visualization methods for logic language are not clear on basic static and dynamic features, creating a conceptual gap that can lead to misinterpretation [18-21]. Accordingly, an FM-based representation can be used to schematize logic formulas, i.e., express or reduce them to a diagram. A logical formula such as $p$ indicates a sphere with two types of flowthings: $p$, and Truth/Falsity values. Thus, $p$ can be conceptualized as a sphere $p$ formed from two subsystems: Body (symbolic expression), and Truth, as shown in Fig. 5. Such an idea has been introduced before and used in describing the flow of truth values among formulas. Note that the body of $p$ as a sequence of symbols is a thing that can be created, transferred, released, received, and processed.

The new contribution in this paper is application of FM diagrams to meaning (sense), as will be discussed in the next two sections.

$$
p \quad \text { Body } \quad \text { Truth/Falsity values }
$$

Fig. 5. $\quad p$ as a sphere with two flowsystems

\section{DiAgRAMMATIC REPRESENTATION OF FREGE's SENSE}

For Gottlob Frege (1848-1925), a founding father of modern logic, the notion of meaning comprised two aspects:

1) A reference (referent), the thing an expression refers to.

2) A sense, the way such an expression refers to that object.

According to Frege, every linguistic expression denotes its reference, and expresses its sense. For example, the sense of desk is that of a piece of furniture with a flat top and legs, used for reading and writing. As in this example, sense can indicate meaning by expression in a natural language (e.g., words in a dictionary).
As a diagrammatic language, FM provides an alternative representation of the sense of an expression. Since we have already seen that FM expresses body and truth values, the same can be applied to illustrate sense and thus provide a uniform diagrammatic representation of the three aspects of a formula: body, truth value, and sense. Consider the following example:

For Frege, the names morning star and evening star reflect different senses of the same referent: the physical star, Venus. There had been a time when this correspondence was unknown and the two stars were thought to be different; now, the two terms have the same meaning since they refer to the same physical planet. In this case, the sense is an implicit understanding of the meaning based on context.

[Frege] called the sense of a term the "mode of presentation" of the referent. So while "the Morning Star" and "the Evening Star" both refer to the same thing, they have different senses: the sense of "the Morning Star" is something like,

1) The bright star that rises in the early morning,

while the sense of "the Evening Star" is something like,

\section{2) The bright star that rises in the early evening}

Same reference; different sense. [23] (numbers and italics added; for details of this problem, see [24])

Accordingly, FM representation can be applied to these sentences in terms of the body (red in the online version of the paper), truth value, and sense, as shown in Fig. 6. In the upper diagram, The bright star that rises in the early evening, the referent (circle 1), the bright (2) star, is described in the sense of having been created - making its first appearance, rising in the early (3) morning (4). Here the term Create denotes first existence or appearance in the total context. Note that morning, earliness, star, brightness, and sense are all (flow) things. Creation is a type of flow (from nonexistence). 


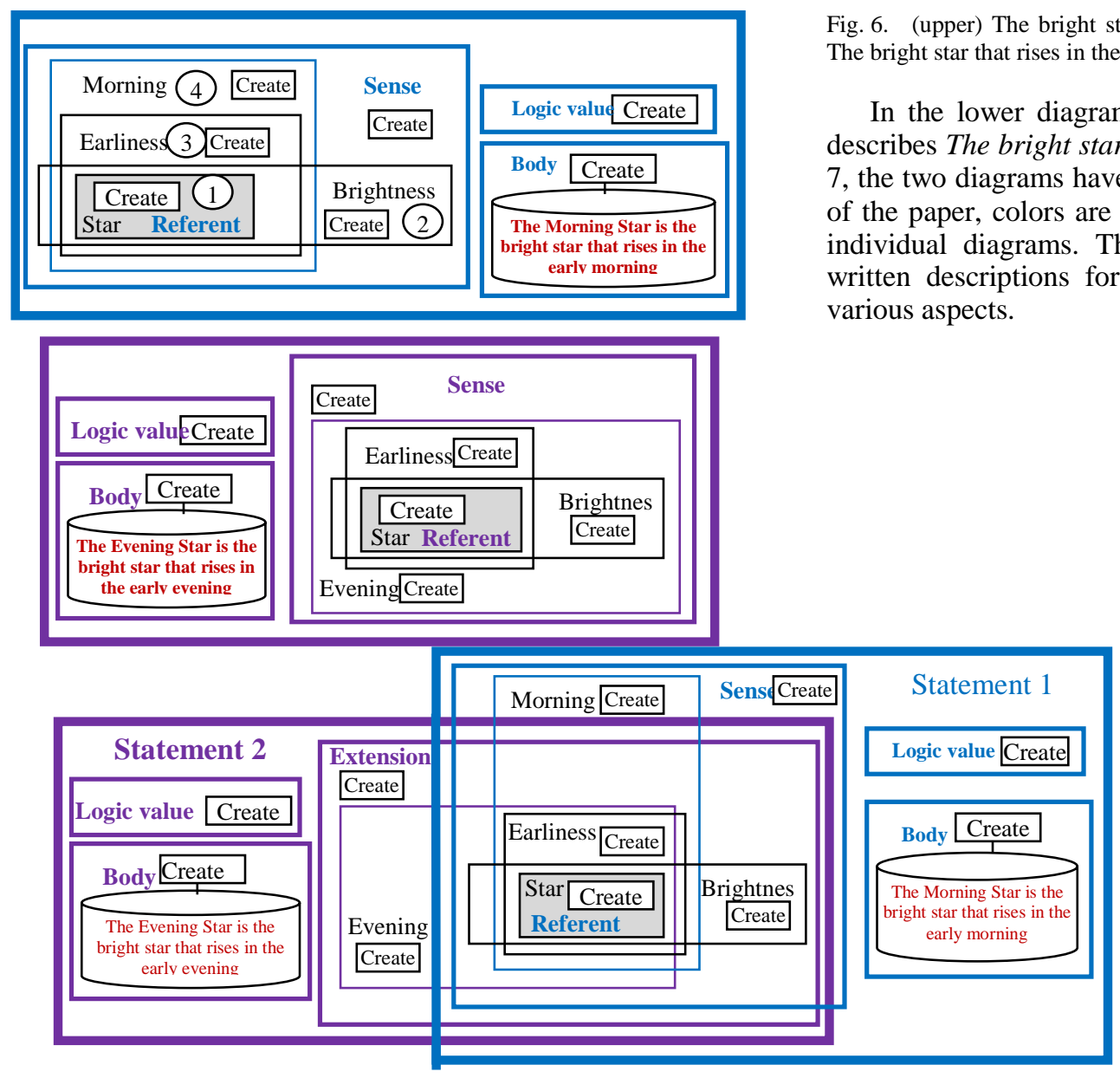

Fig. 7. The bright star that rises in the early morning and The bright star that rises in the early evening

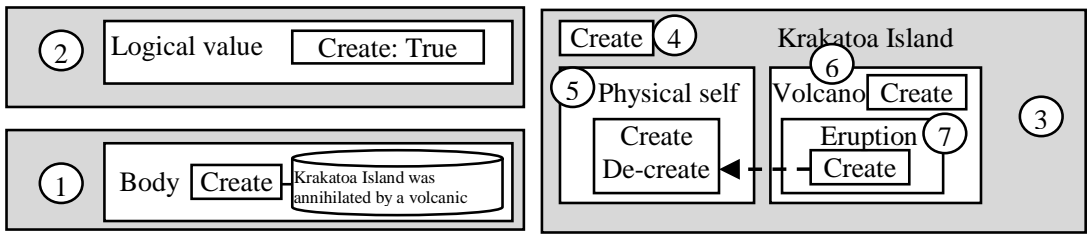

Fig. 8. It is true that Krakatoa Island was annihilated by a volcanic eruption if and only if Krakatoa Island was annihilated by a volcanic eruption

\section{REPRESENTING POSSIBLE WORLDS}

Bradley and Swartz [25] considered that, for any item and any attribute $a$ and $F$, we can define "truth" and "falsity" as follows:

\section{a) It is true that a has $F$ if, and only if, a has $F$}

For example, It is true that Krakatoa Island was annihilated by a volcanic eruption if and only if Krakatoa Island was annihilated by a volcanic eruption.

This can be represented by FM diagram as shown in Fig. 8. In such a world, there are three spheres: the Proposition (1), its truth value (2), and its meaning (3). The stage of Create in 1, 2, and 3 specifies existence. The meaning includes the Island sphere (4) in terms of its subspheres: its physical self (5) and the Volcano (6) that contains the sub-subsphere, Eruption (7). Create and De-create express that at the beginning there exists, physically, an island and because of the eruption, it no longer exists. Note that we do not differentiate between a proposition and a sentence in this example (see [25]).

Bradley and Swartz [25] describe the possible states of the elements as follows:

1) Krakatoa Island exists but fails to have the attribute annihilated by a volcanic eruption

In this case, the fragment annihilated by a volcanic eruption in the sentence is a fiction. Accordingly, we have the two worlds Real and Fiction, as shown in Fig. 9. The island exists in reality (circle 1), but the volcano (2) and the destruction (3) of the island exist in fiction. In reality, the body of symbols Krakatoa Island was annihilated by a volcanic eruption exists in reality (4). The whole proposition is False in reality (5), but it is true in fiction (6), e.g. a novel. 


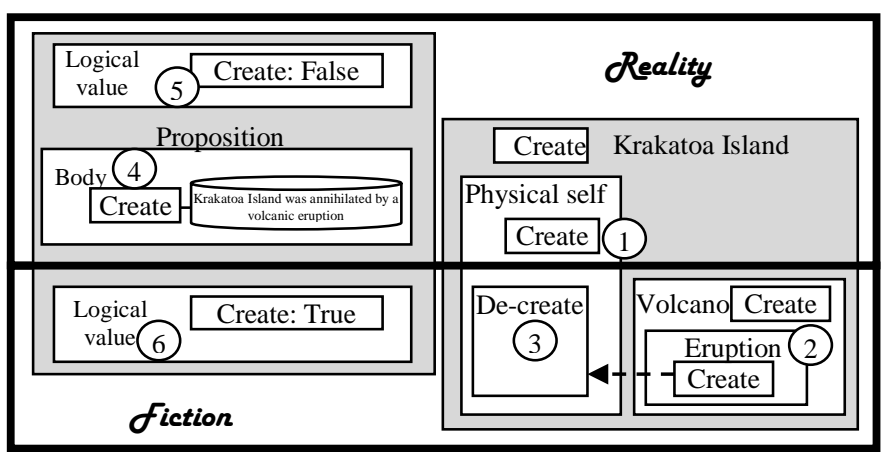

Fig. 9. Krakatoa Island exists but lacks the attribute annihilated by a volcanic eruption

2) Krakatoa Island does not exist. Since an attribute can be instanced by a logical item in a possible world only if that item exists in that possible world, the failure of an item to exist in a given possible world precludes it from having any attributes whatever in that world. [25]

Fig. 10 shows the corresponding FM representation. Krakatoa Island was annihilated by a volcanic eruption is just a sequence of symbols. It is False in reality; however, it has fictitious meaning and is True in the context of fiction, e.g., in a movie.

These notions are viewed as things that can be created, processed, released, transferred, and received. For example, the meaning of the words Krakatoa Island was annihilated by a volcanic eruption could be created in the sphere of the mind of a news correspondent who then processes this meaning, triggering creation of the news: Krakatoa Island was annihilated by a volcanic eruption in linguistic form (body); accordingly, the news is printed in a newspaper that is read by a person, thus creating a meaning in his/her mind with the truth value: True.

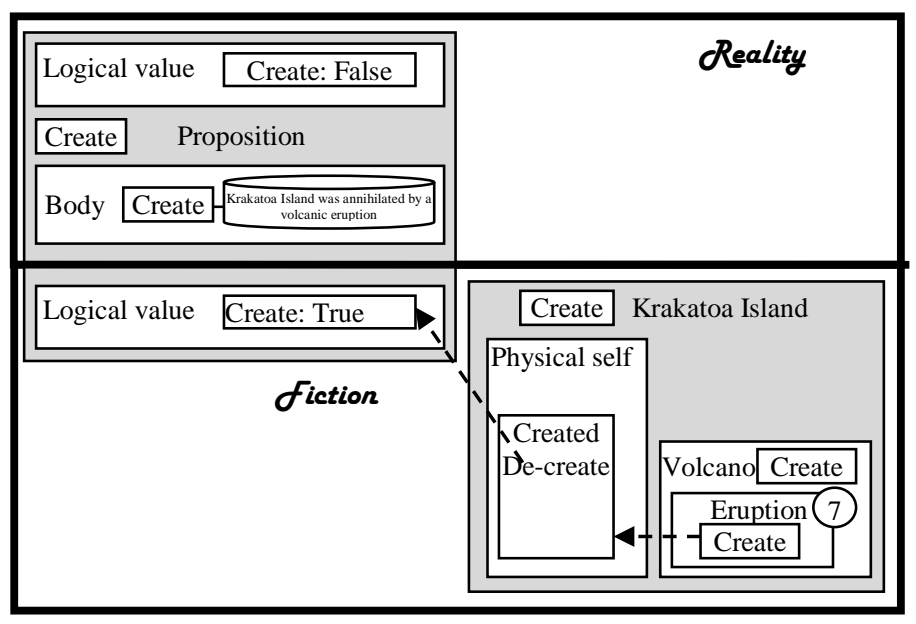

Fig. 10. Krakatoa Island does not exist

\section{CONCLUSION}

This paper has proposed a methodology for mapping the sense of a logic formula through a schema that connects a linguistic conception, truth-values, and meaning in a unifying diagrammatic apparatus. Since diagrams have proven useful for enhancing understanding in design, and as a tool for teaching, the description produced can be especially useful for students specializing in logic representation and reasoning.

It is important to note that this paper is not a contribution in the area of logic. Rather, it has utilized a diagrammatic methodology for flowcharting logic formulas, i.e., expressing them in terms of linguistic expression (body), truth-value, and sense. The diagrams are depictive illustrations that can be used to help students see the relationships among symbolic formulas, associated truth values, and meanings. The method is similar to drawing a flowchart of a computer program that represents the program but does not replace it. FM could potentially be applied in "diagrammatic thinking," and in diagrammatic representations used in artificial intelligence.

The contribution of this paper is limited to proposing use of the diagramming methodology and demonstrating its viability for representing certain situations. A great deal of material has been left for future work, including quantifiers and adduction. Nevertheless, the FM representation is worth further discussion and investigation for its advantages in illustrating of formulas and their relationships. Furthermore, at this point, this proposed representation of logic formulas does not imply their use in any process such as proofs and reasoning.

Currently, the FM-based description is used as an aid in teaching a discrete structures course in computer engineering. Initial observations indicate mixed results in an academic setting; hence, the model is worth further discussion and investigation for portraying problems in such topics as logic and set theory.

\section{REFERENCES}

[1] T. G. Gill, "Teaching flowcharting with FlowC," Journal of Information Systems Education, vol. 15, no. 1, pp. 65-77, 2004.

[2] D. A. Scanlan, "Structured flowcharts outperform pseudocode: an experimental comparison,” IEEE Software, pp. 28-35, Sept. 1989.

[3] T. Crews and J. Butterfield, "Using technology to bring abstract concepts into focus: a programming case study," Journal of Computing in Higher Education, vol. 13, no. 2, pp. 25-50, 2002.

[4] T. Brooks, The Mythical Man-Month: Essays on Software Engineering Reading, MA: Addison Wesley, 1975.

[5] B. Shneiderman, R. Mayer, D. McKay, and P. Heller, "Experimental investigations of the utility of detailed flowcharts in programming." Communications of the ACM, vol. 20, no. 6, pp. 373-381, 1977.

[6] G. Kesteven, "Flowcharts make bad documentation" [blog post], April 29, 2015. http://phrontex.com/blog/?p=164

[7] S.-J. Shin, O. Lemon, and J. Mumma, "Diagrams," Stanford $\begin{array}{lll}\text { Encyclopedia } & & \end{array}$ http://plato.stanford.edu/entries/diagrams/

[8] A. Shimojima, "The graphic linguistic distinction," Artificial Intelligence Review, vol. 13, pp. 313-335, 2001.

[9] K. Stenning, "Distinctions with differences: comparing criteria for distinguishing diagrammatic from sentential systems," in Diagrams 2000, M. Anderson, P. Cheng, and V. Haarslev, Eds. LNCS (LNAI), vol. 1889, pp. 132-148, 2000.

[10] R. Arnheim, Visual Thinking. Berkeley: University of California Press, 1980.

[11] A. Barr and E. A. Feigenbaum, The Handbook of Artificial Intelligence, Vol. 1. Los Altos, CA: William Kaufmann, 1981, pp. 200-206.

[12] A. Sloman, "Interactions between philosophy and A.I.: the role of intuition and non-logical reasoning in intelligence," Artificial Intelligence, vol. 2, pp. 209-225, 1971. 
[13] A. Sloman, "Afterthoughts on analogical representations," in Proceeding of the 1st Workshop on Theoretical Issues in Natural Language Processing (TINLAP-1), Cambridge, MA, 1975, pp. 164-168.

[14] Y. Sato, K. Mineshima, and R. Takemura, "Interpreting logic diagrams: a comparison of two formulations of diagrammatic representations," in Proceedings of the 33rd Annual Conference of the Cognitive Science Society, C. Hoelscher, T. F. Shipley, and L. Carlson, Eds. Austin, TX: Cognitive Science Society, 2011, pp. 2182-2187.

[15] S.-J. Shin, The Logical Status of Diagrams. Cambridge University Press, 1994.

[16] S. Al-Fedaghi, "Anatomy of personal information processing: application to the EU privacy directive," International Journal of Liability and Scientific Enquiry, vol. 1, no. 7, 2007.

[17] C. Borysowich, "Using logic flowcharts," Toolbox.com, Apr. 27, 2007. http://it.toolbox.com/blogs/enterprise-solutions/using-logic-flowcharts15950

[18] S. Al-Fedaghi, "Schematizing proofs based on flow of truth values in logic," IEEE International Conference on Systems, Man, and Cybernetics (IEEE SMC 2013), Manchester, UK, 2013.
[19] S. Al-Fedaghi, "Visualizing logical representation and reasoning," 15th International Conference on Artificial Intelligence (ICAI'13), Las Vegas, NV, USA, 2013.

[20] S. Al-Fedaghi, "How to diagram your logical argument," Intelligent Systems Conference 2015 (IntelliSys 2015), London, 2015.

[21] S. Al-Fedaghi, "Schematizing formulas for logic students," International Journal of Intelligent Information Processing, vol. 4, no. 4, pp. 27-38, 2013.

[22] S. Al-Fedaghi, "Function-behavior-structure model of design: an altenative approach," International Journal of Advanced Computer Science and Applications, vol. 7, no. 7, pp. 133-139, 2016.

[23] A. Julien, "Frege was from Venus" [blog post], June 20, 2013. https://welovephilosophy.com/tag/evening-star/

[24] Stanford Encyclopedia of Philosophy, "Gottlob Frege," Sep 13, 2016. http://plato.stanford.edu/entries/frege/

[25] R. Bradley and N. Swartz, "Introduction: Possible Worlds," in Logic and Its Philosophy, Hackett, 2008. 